\title{
High-Sensitivity Sensor Fabricated on the Basis of Single-Mode Fiber Loop Using Frequency-Sweeping-Generated Ringing Effect
}

\author{
Yundong Zhang*, Ying Wang, Yulong Gai, Xuenan Zhang and Ping Yuan \\ Institute of Opto-Electronics and National Key Laboratory of Tunable Laser Technology, \\ Harbin Institute of Technology, Harbin 150080, China
}

(Received November 5, 2012; accepted February 4, 2013)

Key words: ringing phenomenon, frequency sweeping, optical fiber resonator, detector

Currently, people are looking for a simple and effective method to detect minor changes in fluid environments. In this article, we propose a simple method to detect such changes using the ringing phenomenon generated by frequency sweeping in a single-mode optical fiber resonator. The method is based on the fact that the insertion loss of the optical fiber resonator is determined by environmental changes alone, which leads to the changes in the cavity ringdown time of the fiber resonator. It has several superior advantages over the frequency-domain method, one of which is that the minimal detectable optical loss is on the order of $10^{-6}$. In this article, we also show that the sensing system is insensitive to temperature drifting, which overcomes the main defect of fiber loop resonators, that is, they are extremely sensitive to temperature changes. The structure of the sensor is compact and stable owing to the characteristic of optical fiber resonators, and it is easy to use in practical application because the structure is not delicate and is easy to construct.

\section{Introduction}

In modern medical and biological fields, there is a need to detect, measure, and determine minute changes in the human body, microfluids, or other liquid environments, which require the development of safe high-precision sensing techniques.

Optical devices, particularly fiber devices, have been widely used for sensing, owing to the fact that they are ultrasensitive to outside changes in anti-electromagnetic interference, corrosion resistant, capable of covering long distances, multiplexible, small, low cost, and light weight. Moreover, they are particularly suitable for use in the medical and biological fields because they have little impact on detection media.

Examples of such optical devices are the Fiber-Bragg-grating-based sensor, ${ }^{(1)}$ macrobending single-mode fiber-based refractometer, ${ }^{(2)}$ tapered fiber-based sensor, ${ }^{(3)}$

*Corresponding author: e-mail: ydzhang@hit.edu.cn 
and optical-ring-resonator-based sensor. ${ }^{(4)}$ However, all these devices have problems. Despite their high sensitivity $\left(5.23 \times 10^{-6} \mathrm{RIU}\right)$, Fiber-Bragg-grating-based sensing heads are delicate optical devices, which are difficult to manipulate and are easily damaged. Optical ring resonators should have a large quality factor to increase their capability, since they primarily detect the wavelength shift $\Delta \lambda$. Thus, the usual way to realize this is to decrease the size of the resonators to the size of microfiber coil resonators, which are also difficult to fabricate and manipulate and are easily damaged. These abovementioned defects make them difficult to apply to practical circumstances.

Over the past twenty years, cavity ring-down spectroscopy, which was originally developed for quantifying high-reflectivity mirrors, has been widely used in many fields, ${ }^{(5)}$ such as wavelength calibration, ${ }^{(6)}$ harmful substance detection, ${ }^{(7)}$ and environmental atmosphere monitoring. ${ }^{(8)}$ Because of the multipass nature of the optical absorption path, it easily obtains high sensitivity. The fiber loop ring-down technique, which has been proved useful for chemical and physical quantity sensors, such as gas or liquid sensors, ${ }^{(7)}$ temperature sensors, ${ }^{(9)}$ strain sensors, ${ }^{(10)}$ and refractive index sensors, ${ }^{(11)}$ has evolved by combining cavity ring-down spectroscopy and optical fiber technology. Most of these sensors are excited by a pulse laser. By monitoring the vibration and disappearance of a pulse injected into the fiber ring resonator, we can observe the changes in loss parameter in the resonator, and then the changes in the outside environment, which lead to these changes.

In this report, we demonstrate that a novel method to determine the coupling regime of high-Q resonators - ringing phenomenon - can also be used to detect environmental changes. It also has the characteristics of high accuracy and nonrequirement of a laser with high stable frequency. Moreover, we prove that the main defects of conventional ring-resonator-based sensors, which are sensitive to temperature drifting owing to the fact that the length and effective index of the resonator change with temperature variations, can be overcome by this method.

\section{Materials and Methods}

The system we used was a ring resonator with the resonant angular frequency $\omega_{0}$ side-coupled to a waveguide ${ }^{(12)}$ as in Fig. 1. The coupling between the resonator and waveguide can be described by the lifetime $\tau_{e}$. The intrinsic lifetime of the resonator $\tau_{0}$ characterizes the loss of the waveguide in the resonator, and $\tau$ represents the total loss of the resonator in the system. The temporal evolution of the mode amplitude $u$ in the resonator can be described on the basis of the coupled-mode theory in time:

$$
\frac{\mathrm{d} u}{\mathrm{~d} t}=\left(j \omega_{0}-\frac{1}{\tau}\right) u(t)+\sqrt{\frac{2}{\tau_{\mathrm{e}}}} S_{\mathrm{in}}(t),
$$

where $S_{\text {in }}(t)$ is the input field. The relation between the waveguide output field $S_{\text {out }}$ and $u$ is

$$
S_{\text {out }}(t)=-S_{\text {in }}(t)+\sqrt{\frac{2}{\tau_{\mathrm{e}}}} u(t),
$$




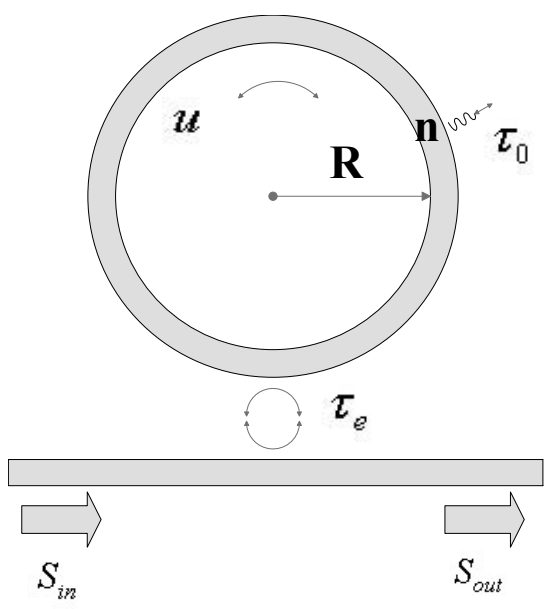

Fig. 1. Schematic of the static resonator configuration.

In the above formulas, $\tau$ is the lifetime describing the total loss of the system, which is given by

$$
\frac{1}{\tau}=\frac{1}{\tau_{\mathrm{e}}}+\frac{1}{\tau_{0}} .
$$

Therefore, once we determine the input field $S_{\text {in }}(t)=S_{0} \exp \left[j\left(\omega_{i} t+V_{\mathrm{s}} t^{2}\right)\right]$, the mode propagation in the system is entirely dependent on the lifetimes of the resonator. The coupling lifetime $\tau_{\mathrm{e}}$ and intrinsic lifetime $\tau_{0}$ are related to the intensity coupling coefficient $\kappa$ and intensity roundtrip attenuation $a$, respectively,

$$
\begin{gathered}
\tau_{\mathrm{e}}=\frac{2 n L}{c \kappa^{2}}, \\
\tau_{0}=\frac{2 n L}{c\left(1-a^{2}\right)}=\frac{2 n L}{c A},
\end{gathered}
$$

where $n$ is the effective group index of the mode and $L$ is the roundtrip length propagation and $A=1-a^{2}$.

\section{Results}

To fabricate the structure, we used a resonator with a cavity length of $5 \mathrm{~m}$, and then stripped a small section of the fiber cladding to form the sensing head, because the bare fiber is so small that it will not change the effective group index $n$ of the resonator. 
When we put the bare fiber into the fluid that we wanted to examine, the total optical loss will vary because the fluid changes the evanescent field of the resonator.

We already know that the ringing phenomenon will appear when the frequency sweeping speed $V_{\mathrm{S}}$ reaches the order of $1 / \tau^{2}$. When the only variation of the system is optical loss $A$, we can see the ringing profile with different transmission losses as shown in Fig. 2. We asumme the same resonator with $L=5 \mathrm{~m}, n=1.5$, and $V_{\mathrm{S}}=1 / \tau^{2}$.

We can see that the changes in intrinsic loss will lead to a markedly significant difference between these ringing profiles. To detect the loss changes quantitatively, we propose the use of exponential fitting to link the lowest points of the ringing profiles $A_{1} A_{2} \cdots A_{n}$ as shown in Fig. 3, and then we can find the time $t_{\mathrm{B}}$, which conform to $\left|1-t_{\mathrm{B}}\right|$ $=\left|1-t_{\mathrm{A}}\right| \times e^{-1}$. By recording $\Delta t=t_{\mathrm{A}_{1} \mathrm{~B}}$, we can obtain the changes in additional optical transmission loss, then the refractive index changes of the fluid.

\section{Discussion}

As we already know, ${ }^{(3)}$ the minimum detectable optical loss is $B_{\min }=(1 / m)\left(\sigma_{\tau} / \bar{\tau}\right), \sigma_{\tau}$ is the standard deviation of the ringdown time, $\bar{\tau}$ is the average ringdown time, and $\sigma_{\tau} / \bar{\tau}$ is the ringdown baseline noise, which is on the same order of $10^{-3}$ as the minimum detectable change in light intensity $\Delta I / I_{0}$, in a conventional intensity-based sensing scheme. The improvement factor $m$, which is the number of round trips of light within the ringdown time $\tau_{0}$, can be described as $m=\mathrm{c} \tau_{0} / n L$, which in our structure is on the order of $10^{3}$, so the minimum detectable optical loss is on the order of $10^{-6}$.

Moreover, we discover that the method is insensitive to the length of the resonator. By comparing the detection time $\Delta t$ between two different resonators with $L=5 \mathrm{~m}$ and $L$ $=4 \mathrm{~m}$, respectively, as shown in Fig. 4, we can reach the above-mentioned conclusion.

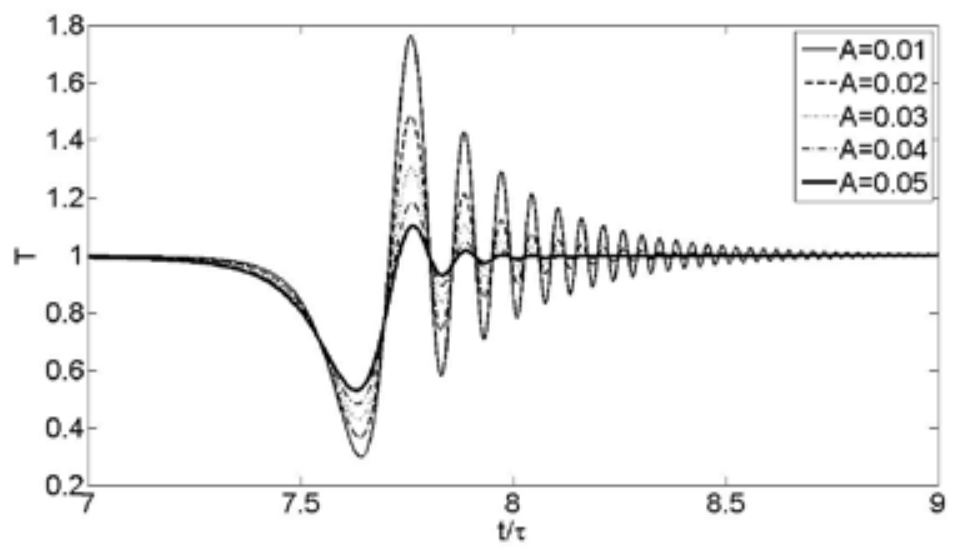

Fig. 2. Ringing profile of the resonator vs various intrinsic losses $A$. Here, we set $A$ equals 0.01 to 0.05 . 


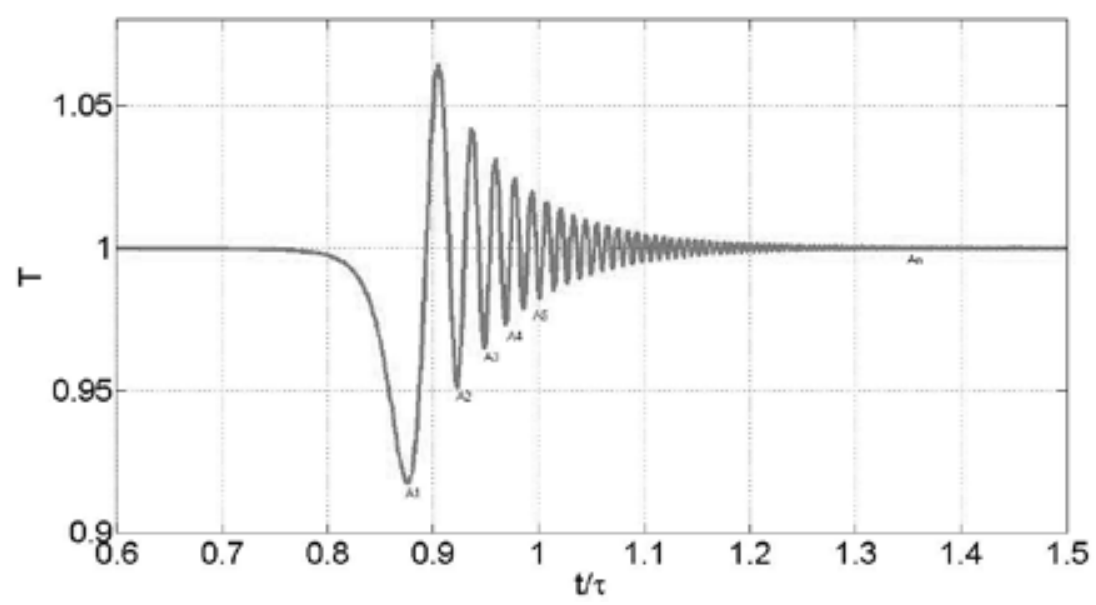

Fig. 3. Ringing profile of a set system. $A_{1} \cdots A_{n}$ are the points we chose to describe the profile.

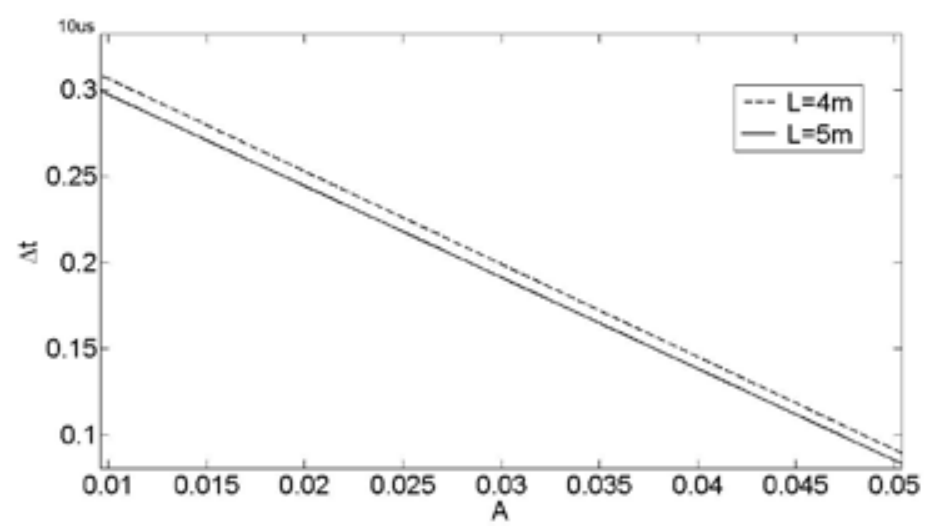

Fig. 4. Ringdown time $\Delta t$ vs the intrinsic loss $A$. Here, we set the resonator lengths to be $4 \mathrm{~m}$ (dashed line) and $5 \mathrm{~m}$ (solid line).

In Fig. 4, the red curve represents the condition of $L=4 \mathrm{~m}$ and the blue curve represents $L=5 \mathrm{~m}$. We can see a resonator length difference of $\Delta L=1 \mathrm{~m}$, and $\Delta t$ is negligible at the same $A$, which has a fluctuation of $1.6 \%$ when $A=0.01$. Thus, we can conclude that $\Delta t$ is primarily determined by transmission loss. Therefore, under normal circumstances, this method is insensitive to temperature changes, and we can avoid using microspheres, microdisks, or microrings as detection heads, which are all delicate and difficult to fabricate. 


\section{Conclusions}

We have demonstrated that ringing phenomenon in the single-mode fiber resonator generated by fast frequency sweeping can be used as a sensor to test the minor changes in the refraction index of a fluid environment. The method has several superior advantages over the frequency-domain method. We have also shown that a small change in total transmission loss can lead to a marked variation in transmission spectroscopy. Most importantly, the system is insensitive to temperature changes, which overcomes the main defect of fiber loop resonators, that is, they are extremely sensitive to temperature drifting.

\section{Acknowledgements}

The study was supported by the National Natural Science Foundation of China under Grants Nos. 61078006 and 60878006.

\section{References}

1 Q. Wu, Y. Semenova, B. Yan, Y. Ma, P. Wang, C. Yu and G. Farrell: Opt. Lett. 36 (2011) 2197.

2 P. Wang, Y. Semenova, Q. Wu, G. Farrell, Y. Ti and J. Zheng: Appl. Opt. 48 (2009) 6044.

3 P. Wang, G. Brambilla, M. Ding, Y. Semenova, Q. Wu and G. Farrell: Opt. Lett. 36 (2011) 2233.

4 I. M. White, H. Oveys and X. Fan: Opt. Lett. 31 (2006) 1319.

5 D. Z. Anderson, J. C. Frisch and C. S. Masser: Appl. Opt. 23 (1984) 1238.

6 Z. Tan, X. Long, J. Yuan, Y. Huang and B. Zhang: Appl. Opt. 48 (2009) 2344.

7 C. Ramos and P. J. Dagdigian: Appl. Opt. 46 (2007) 620.

8 D. S. Baer, J. B. Paul, M. Gupta and A. O'Keefe: Appl. Phys. B: Lasers Opt 75 (2002) 261.

9 C. Wang: in Technical Digest (CD) (Optical Society of America, Washington, DC, 2006), PWA2.

10 J. Gan, Y. Hao, Q. Ye, Z. Pan, H. Cai, R. Qu, and Z. Fang: Opt. Lett. 36 (2011) 879.

11 C. Wang and C. Herath: Opt. Lett. 35 (2010) 1629.

12 S. Trebaol, Y. Dumeige and P. Féron: Phys. Rev. A: At. Mol. Opt. Phys. 81 (2010) 043828. 